\title{
Into another dimension: Assessing novel therapeutics in a more lifelike microenvironment
}

\author{
Mara B. Antonoff, MD
}

See related article on pages 1417-25.

In 2011, Colson and colleagues ${ }^{1}$ published their experiences with paclitaxel-loaded expansile nanoparticles (Pax-eNPs), demonstrating significant antitumor efficacy against mesothelioma both in vitro and in a murine model in vivo. Although this study importantly showed that Pax-eNP delivery was more efficacious than conventional drug delivery (Pax-C/E) in vivo, such superiority of this delivery system was not predicted by the in vitro experiments with monolayer cell cultures. In a follow-up article in this issue of the Journal by Lei and colleagues, ${ }^{2}$ through a series of elegant and well-thought out experiments, the group utilized an alternative in vitro model, finding that a 3 -dimensional spheroid more closely replicated the environment of the in vivo xenograft model, with similar superior efficacy of Pax-eNPs. Further, Lei and colleagues ${ }^{2}$ sought to characterize the mechanisms of enhanced antitumor activity, identifying macropinocytosis as the dominant endocytotic pathway of expansile nanoparticle uptake. While this study contributes greatly to the body of knowledge available regarding potential treatment strategies for malignant mesothelioma, one might argue that the more important impact of this article relates to the successful implementation of an unconventional tumor model.

In their earlier studies, these authors acknowledged the limitations of the commonly used 2-dimensional cell monolayer as a template for in vitro experiments. Recognizing the palpable knowledge gap and the need for a cost-effective, accurate in vitro model, they aimed to find a solution that better approximates in vivo biologic behaviors without the limitations and cost of animal models.

Molecular and genetic mechanisms underlying a variety of malignancies have been extensively studied in 2dimensional models with cultured cancer cells growing as a monolayer. Unfortunately, despite the massive volume of

From the Department of Thoracic and Cardiovascular Surgery, University of Texas MD Anderson Cancer Center, Houston, Tex.

Disclosures: Author has nothing to disclose with regard to commercial support.

Received for publication Feb 9, 2015; accepted for publication Feb 9, 2015; available ahead of print March 17, 2015.

Address for reprints: Mara B. Antonoff, MD, Department of Thoracic and Cardiovascular Surgery, University of Texas MD Anderson Cancer, 1400 Pressler St, Unit 1489, Houston, TX 77041 (E-mail: mbantonoff@mdanderson.org).

J Thorac Cardiovasc Surg 2015;149:1426-7

$0022-5223 / \$ 36.00$

Copyright (c) 2015 by The American Association for Thoracic Surgery

http://dx.doi.org/10.1016/j.jtcvs.2015.02.015 literature based on such studies, these models cannot perfectly mimic the native cellular environment, nor do they represent accurate 3-dimensional cellular morphology. ${ }^{3}$ While in vivo models successfully provide a realistic biologic microenvironment in 3 dimensions, these models are not without limitation either. The commonly utilized human tumor

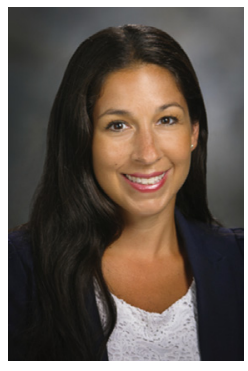
xenograft involves injection of human cancer cells into an immunocompromised animal, typically a mouse, and allowing the tumor to grow before conducting therapeutic interventions and/or investigations. ${ }^{4}$ Live animal models represent a more realistic standard, but they can be expensive to purchase and maintain, limiting study sizes per experiment. Further, assessment of treatment efficacy can be a challenge, requiring animal sacrifice or complex imaging that may be cost-prohibitive.

Cancer cell spheroids are 3-dimensional cell colonies functioning in an artificially created environment, allowing cells to engage in more realistic cell-cell and cell-matrix interactions within the microenvironment. ${ }^{5}$ These culture models provide a compromise between the unrealistic 2-dimensional monolayer and the complex xenogeneic host animal. ${ }^{3}$ Although they have 3 dimensions, spheroid models still rely on simple diffusion for nutrients and oxygen, which limits the size of cell aggregate that may be useful; however, this type of nutrient restriction may actually better recapitulate tumor microenvironment than a cell monolayer bathing in nutrients.

Spheroids recreate many of the physiologic parameters present in vivo, including complex multicellular architecture, extracellular matrices, and obstacles that limit mass transport. Such aspects allow spheroids to serve as a significantly more realistic and physiologic platform for testing novel therapeutics as compared to 2-dimensional monolayers. Widespread adoption is somewhat limited, at present, due to the complexities of spheroid formation, requiring time and expertise; nonetheless, it is clear that such models are a huge improvement over our current in vitro models.

In this study, Lei and colleagues ${ }^{2}$ offer a mechanistic explanation for their observations of improved survival in vivo after treatment with Pax-eNP compared with free paclitaxel, despite the absence of such findings in 2-dimensional, monolayer in vitro studies. Detailed experiments helped elucidate cytotoxic mechanisms and nanoparticle-tumor interactions, which are of pertinent 
clinical relevance in terms of paclitaxel delivery. Moreover, the utilization of a 3-dimensional model aids in elevating the standard for investigators studying a multitude of tumor types, with a breadth of potential therapeutic interventions. The benefits offered by the spheroid model are simple yet critical. For any of our studies to be relevant, they must have validity in terms of representing the true biologic environment of human patients. With such cutting-edge studies, this group encourages us all to continue to seek models that are translatable, so that we may move closer to our ultimate goal: curing cancer in real, live people.

There are a few limitations of the study, which are appropriately identified by Lei and colleagues. ${ }^{2}$ The 3-dimensional spheroid model may lack the multiple cell types typically seen in actual tumors. Further, quantitative assessment may be a challenge in this model. However, these studies have clearly shown innovation, and they serve as an example for all investigators looking to impact cancer. The ability to more accurately assess in vivo behaviors with an in vitro model provides a platform demonstrating responsibility in terms of financial cost and resource utilization. Further, improving our ability to predict in vivo activity allows enhanced screening of novel drugs and drug-delivery systems, thus benefiting our patients enormously. This timely and well-constructed study provides a foundation upon which we may build our knowledge of chemotherapeutic delivery mechanisms while setting an outstanding example, and perhaps a new standard, for in vitro methodology.

\section{References}

1. Colson YL, Liu R, Southard EB, Schulz MD, Wade JE, Griset AP, et al. The performance of expansile nanoparticles in a murine model of peritoneal carcinomatosis. Biomaterials. 2011;32:832-40.

2. Lei H, Hofferberth SC, Liu R, Colby A, Tevis KM, Catalano P, et al. Paclitaxel-loaded expansile nanoparticles enhance chemotherapeutic drug-delivery in mesothelioma 3D multicellular spheroids. J Thorac Cardiovasc Surg. 2015;149:1417-25.e1.

3. Nyga A, Cheema U, Loizidou M. 3D tumour models: novel in vitro approaches to cancer studies. J Cell Commun Signal. 2011;5:239-48.

4. Morton CL, Houghton PJ. Establishment of human tumor xenografts in immunodeficient mice. Nat Protoc. 2007;2:247-50.

5. Pampaloni F, Reynaud EG, Stelzer EH. The third dimension bridges the gap between cell culture and live tissue. Nat Rev Mol Cell Biol. 2007;8:839-45. 